\title{
A comparative study of machine learning and deep learning algorithms to classify cancer types based on microarray gene expression data
} \author{
Rodriguez-Sotelo ${ }^{1}$, Cristian Felipe Jiménez Varón ${ }^{6}$ \\ ${ }^{1}$ Department of Electronics and Automation, Universidad Autónoma de Manizales, Manizales, Caldas, Colombia \\ 2 Department of Computer Science, Universidad Autónoma de Manizales, Manizales, Caldas, Colombia \\ 3 Department of Systems and informatics, Universidad de Caldas, Manizales, Caldas, Colombia \\ 4 Department of Automatics and Electronics, Universidad Autónoma de Occidente, Cali, Valle del Cauca, Colombia \\ 5 Izmir International Biomedicine and Genome Institute, Izmir, Turkey \\ 6 Department of Physics and Mathematics, Universidad Autónoma de Manizales, Manizales, Caldas, Colombia \\ Corresponding Authors: Reinel Tabares-Soto, Simon Orozco-Arias \\ Email address: rtabares@autonoma.edu.co, simon.orozco.arias@gmail.com
}

Cancer classification is a topic of major interest in medicine since it allows accurate and efficient diagnosis and facilitates a successful outcome in medical treatments. Previous studies have classified human tumors using a large-scale RNA profiling and supervised Machine Learning (ML) algorithms to construct a molecular-based classification of carcinoma cells from breast, bladder, adenocarcinoma, colorectal, gastro esophagus, kidney, liver, lung, ovarian, pancreas, and prostate tumors. These datasets are collectively known as the 11_tumor database, although this database has been used in several works in the ML field, no comparative studies of different algorithms can be found in the literature. On the other hand, advances in both hardware and software technologies have fostered considerable improvements in the precision of solutions that use $\mathrm{ML}$, such as Deep Learning (DL). In this study, we compare the most widely used algorithms in classical $M L$ and DL to classify the tumors described in the 11_tumor database. We obtained tumor identification accuracies between $90.6 \%$ (Logistic Regression) and $94.43 \%$ (Convolutional Neural Networks) using k-fold cross-validation. Also, we show how tuning process may or may not significantly improve algorithms' accuracies. Our results demonstrate an efficient and accurate classification method based on gene expression (microarray data) and ML/DL algorithms, which facilitates the prediction of the type of tumor from a multi-cancer-type scenario. 


\section{A comparative study of machine learning and deep} 2 learning algorithms to classify cancer types based on 3 microarray gene expression data

Reinel Tabares-Soto ${ }^{1}$, Simon Orozco-Arias ${ }^{2,3}$, Victor Romero Cano ${ }^{4}$, Vanesa Segovia Bucheli ${ }^{5}$, Jose Luis Rodriguez-Sotelo ${ }^{1}$, Cristian Jiménez-Varón ${ }^{6}$

${ }^{1}$ Department of Electronics and Automation, Universidad Autónoma de Manizales, Manizales, 9 Colombia.

$10{ }^{2}$ Department of Computer Science, Universidad Autónoma de Manizales, Manizales, Colombia.

${ }^{3}$ Department of Systems and Informatics, Universidad de Caldas, Manizales, Colombia

${ }^{4}$ Department of Automatics and Electronics, Universidad Autónoma de Occidente, Cali, Colombia. Colombia.

Corresponding Authors:

Reinel Tabares-Soto ${ }^{1}$

Antigua estación del ferrocarril, Manizales, Caldas, 170002, Colombia

Email address: rtabares@autonoma.edu.co

Simon Orozco-Arias 2,3

Antigua estación del ferrocarril, Manizales, Caldas, 170002, Colombia

Email address: simon.orozco.arias@gmail.com

\section{Abstract}

Cancer classification is a topic of major interest in medicine since it allows accurate and efficient diagnosis and facilitates a successful outcome in medical treatments. Previous studies have classified human tumors using a large-scale RNA profiling and supervised Machine Learning (ML) algorithms to construct a molecular-based classification of carcinoma cells from breast, bladder, adenocarcinoma, colorectal, gastro esophagus, kidney, liver, lung, ovarian, pancreas, and prostate tumors. These datasets are collectively known as the 11_tumor database, although this database has been used in several works in the ML field, no comparative studies of different algorithms can be found in the literature. On the other hand, advances in both hardware and software technologies have fostered considerable improvements in the precision of solutions that use ML, such as Deep Learning (DL). In this study, we compare the most widely used algorithms in classical ML and DL to classify the tumors described in the 11_tumor database. 
40 We obtained tumor identification accuracies between 90.6\% (Logistic Regression) and 94.43\%

41 (Convolutional Neural Networks) using k-fold cross-validation. Also, we show how tuning

42 process may or may not significantly improve algorithms' accuracies. Our results demonstrate

43 an efficient and accurate classification method based on gene expression (microarray data) and

$44 \mathrm{ML} / \mathrm{DL}$ algorithms, which facilitates the prediction of the type of tumor from a multi-cancer-type

45 scenario.

46

47 Introduction

48 Cancer is one of the most deadly diseases in human health caused by the abnormal proliferation

49 of cells, leading to malignant malformations or tumors with different pathology characteristics

50 (Varadhachary, 2007). Cancer-type classification is critical to increasing patient survival rates.

51 Molecular genetic analyses have discovered genetic alterations, or signatures, with different

52 biological characteristics that allow discerning the responses to several treatments (Greller \&

53 Tobin, 1999). This enables early diagnosis and an accurate treatment; therefore, ensuring the

54 efficacy and reduction of side effects (toxicity) of the treatment (Wang et al., 2005).

55

56

Impaired gene expression is a characteristic of carcinogenic cells (Su et al., 2001). Accordingly,

57

58

59

60

61

62

63

64

65

66

67

68

69

70

71

72

73

74

75

76

77

78

79 microarray gene expression data from tumor cells provide an important source of information to improve cancer in a cost-efficient manner, allowing the use of this strategy in developing countries. Although microarray datasets contain thousands of different genes to be analyzed, an accurate and efficient way of analyzing this amount of data is by Machine Learning (ML) and Deep Learning (DL) algorithms (Motieghader et al., 2017). In particular, these algorithms have been applied in other biological areas, including rules of association (Orozco-Arias et al., 2019). Previous studies demonstrate the use of ML and DL in microarray gene expression to infer the expression of target genes based on landmark gene expression (Chen et al., 2016), in feature selection aimed at finding an informative subset of gene expression (Sharma, Imoto \& Miyano, 2012), and in the diagnosis and classification of cancer types (Fakoor et al., 2013).

A well-known database of gene microarrays related to cancer is the 11_Tumors database (Su et al., 2001), which is available at

https://github.com/simonorozcoarias/ML_DL_microArrays/blob/master/data11tumors2.csv. This dataset is a good example of the curse of dimensionality due to the high number of characteristics and few registers of this database. Therefore, most studies use it to test specific data science techniques, such as feature selection methods (Bolón-Canedo et al., 2014; Wang \& Wei, 2017; Han \& Kim, 2018; Perera, Chan \& Karunasekera, 2018), dimension reduction (Araújo et al., 2011), clustering methods (Sardana \& Agrawal, 2012; Sirinukunwattana et al., 2013; Li et al., 2017), preprocessing techniques (Liu et al., 2019), among others. The 11_Tumors database has also been used in gene selection for cancer classification (Moosa et al., 2016; Alanni et al., 2019). Although the authors achieved high accuracy in these publications, they only used some ML algorithms, one preprocessing strategy, and one learning technique 
80 (supervised or unsupervised), which could add bias to their methodology. Additionally, to date, 81 no comparative study on the application of machine learning in microarray datasets is found in 82 the literature.

83

In several machine learning studies, DL has proven to be a robust technique for analyzing largescale data sets (Bengio, Courville \& Vincent, 2013). With these advances, DL has achieved cutting-edge performance in a wide range of applications, including bioinformatics and genomics (Min, Lee \& Yoon, 2016; Yue \& Wang, 2018), analysis of metagenomics samples (Ceballos et al., 2019), identification of somatic transposable elements in ovarian cancer (Tang et al., 2017), identification and classification of retrotransposons in plants (Orozco-Arias, Isaza \& Guyot, 2019) and cancer classification using Principal Component Analysis (PCA) (Liu, Cai \& Shao, 2011). Recent work by Guillen and Ebalunode demonstrated promising results for the application of DL in microarray gene expression (Guillen \& Ebalunode, 2016).

In general, there are two different tasks that ML algorithms can tackle: supervised and unsupervised learning. In supervised learning, the goal is to predict the label (classification) or response (regression) of each data point by using a provided set of labeled training examples. In unsupervised learning, such as clustering and principal component analysis, the goal is to learn inherent patterns within the data (Zou et al., 2018).

The main goal of any ML task is to optimize model performance not only on the training data but also on additional datasets. When a learned model displays this behavior, it is considered to generalize well. With this aim, the data in a given database are randomly split into at least two subsets: training and validation sets (Zou et al., 2018). Then, a model as complex as possible is learned (training set), tuned (validation set), and tested for generalization performance on the validation set. This process is crucial for avoiding overfitting or underfitting. Therefore, a sound learning algorithm must reach an appropriate balance between model flexibility and the amount of training data. An overly simple model will underfit and make inadequate predictions, whereas an overly flexible model will overfit to spurious patterns in the training data and not generalize (Zou et al., 2018).

In this study, we compare the performance of the most commonly used ML and DL algorithms in bioinformatics (Orozco-arias et al., 2019) in the task of classifying by supervised and unsupervised techniques. We used the 11_Tumor database and applied different preprocessing strategies. Our detailed evaluation and comparison illustrate the high accuracy of these algorithms for tumor identification in a multiple-cancer-type scenario and the influence of preprocessing strategies and tuning processes on these accuracies.

117 
119 ML and DL techniques can learn the characteristics of a given problem from a certain amount of 120 data. These data are usually randomly subdivided into two groups: training and validation. A 121 training data set is used to calibrate the parameters of the model, and a validation data set is 122 utilized for evaluating model performance (Eraslan et al., 2019).

123

124

125

126

127

128

129

130

131

132

133

134

135

136

137

138

139

140

141

142

143

144

145

146

147

148

149

150

151

152

153

154

155

156

157

158
In this paper, we compared results obtained from classifying 11 different tumor classes through different approaches of ML and DL. We began by evaluating two unsupervised methods; the first method is the popular K-means algorithm, in which a given number of prototype samples, also known as cluster centers, are estimated by iteratively assigning data points to prototype samples and updating them as the mean of the assigned samples. The second clustering method tested is hierarchical clustering, which is better suited for irregular shapes than K-means. After, we tested eight different classification algorithms. The most popular one, and the standard baseline in classification problems, is K-Nearest Neighbors (KNN), where classification decisions are done through a voting mechanism and model training stores the dataset in a way that queries can be done efficiently. Another family of classification methods comprises the socalled linear models, for which a learning algorithm estimates as many weights as features from the training data so classification prediction is done as a function of the dot product between the weights and a test sample. Linear models are fast to train, fast to predict, and also scale well to datasets in which the number of features is large compared to the number of samples. The linear methods we tested are Linear Support Vector Classifier (SVC), Logistic Regression (LR), Linear Discriminant Analysis (LDA), Naive Bayesian Classifier (NB), and Multi-Layer Perceptron (MLP).

We also included Decision Tree-methods (DT) such as Random Forests (RF). Unlike linear models, DTs and RFs are invariant to data scaling and work well with features on different scales. Finally, we applied Deep Neuronal Networks (DNN), such as fully connected neural networks, also known as Multi-Layer Perceptron (MLP) and Convolutional Neural Networks (CNNs). MLPs are well-suited for non-linear data, whereas CNNs automatize the costly task of engineering features; an unavoidable task in classical machine learning approaches. The above algorithms are extensively explained in (Michie, Spiegelhalter \& Taylor, 1994; Chollet, 2007).

\section{Datasets}

The datasets used represent measurements of gene expression using cancer microarrays and normal biopsies (Statnikov et al., 2005; Bolón-Canedo et al., 2014), and are consolidated in the "11 Tumors database", which is freely available online at (https://github.com/simonorozcoarias/ML_DL_microArrays/blob/master/data11tumors2.csv). This database consists of 174 samples with 12,533 gene expression microarrays for 11 different types of cancer. The 12,533 microarrays of genetic expression are integers with positive and negative values; these values represent the characteristics that allow the ML and DL algorithms

Peer] Comput. Sci. reviewing PDF | (CS-2019:09:41248:2:0:NEW 13 Feb 2020) 
159

160

161

162

163

164

165

166

167

168

169

170

171

172

173

174

175

176

177

178

179

180

181

182

183

184

185

186

187

188

189

190

191

192

193

194

195

196

197

198

to learn how to classify by cancer type. The types of cancer and the number of patients for each type are shown in table 1 . The classes of each cancer type are unbalanced and remained so in the experimentation.

\section{Preparing the data}

For the experiments, we divided the information into two groups; the first group corresponds to the features $(\mathrm{X})$ and the second group to the classes $(\mathrm{Y})$. The features compose a matrix of size $\mathrm{m}$ $\mathrm{x} \mathrm{n}$ and the classes are a vector of size $\mathrm{n} x 1$, where $\mathrm{m}$ is the number of samples and $\mathrm{n}$ is the number of genes for each class $(12,533)$. The dataset, containing 174 samples, is randomly subdivided into two sub-sets ( $80 \%$ training and $20 \%$ validation), including 139 samples for training and 35 samples for validation. Initial calibration of ML and DL algorithms (training) was done using the training set; then, hyperparameter tuning was performed with the validation set and measured the accuracy of the algorithms. We calculated the accuracy of each algorithm using tuned hyperparameters with $\mathrm{k}$-fold cross-validation and $k=10$ to avoid overfitting.

The dataset used in this paper has the curse of dimensionality since the number of characteristics $(12,533)$ is higher than the number of samples (174) (Powell, 2007). Therefore, the data are dispersed and the results are not statistically stable or reliable, directly affecting the accuracy achieved by ML and DL algorithms. Two preprocessing techniques were used to solve this problem: scaling (Géron, 2017) and principal component analysis (PCA) (Wold, Esbensen \& Geladi, 1987). The first technique guarantees that the data are in a range of suitable values to calibrate the model. With the second technique, the statistical significance is improved and the noise introduced by irrelevant characteristics during model training decreases. In this paper, we worked with several combinations of the preprocessing techniques mentioned above to find the best performance.

Four different datasets were created for the training and validation of each ML or DL algorithm. For the first dataset, we did not apply any preprocessing operations; for the second, we performed a scaling process; for the third, we applied PCA with a retained variance of $96 \%$ to reduce data dimensionality, obtaining a dimensional reduction from 12,533 to 83 features. Finally, for the last dataset, we applied both scaling and PCA, obtaining a dimensional reduction from 12,533 to 113 features (principal components).

\section{Unsupervised learning experiments}

Classification performance is highly correlated with the degree of separability of a dataset; therefore, we analyzed performance using clustering techniques. Based on data labels, we can gain a priori insight into the algorithm that works best on the distribution of the gene expression microarray dataset. 
199

200

201

202

203

204

205

206

207

208

209

210

211

212

213

214

215

216

217

218

219

220

221

222

223

224

225

226

227

228

229

230

231

232

233

234

235

236

237

238

Before applying the classification algorithms (supervised learning), we performed a hierarchical analysis to better understand the dataset. This hierarchical clustering used different distance metrics, such as Ward, average, single, complete, weighted, centroid, and median. Further, as input, we used a dataset with no preprocessing. These distance metrics serve to capture the differences between the data samples and vary in their capacity to deal with large outliers (i.e. between weighted, centroid, and median metrics) or if they allow choosing the number of clusters to consider (e.g. Ward) (Foss, Markatou \& Ray, 2019). After this clustering, we tested all of the datasets created in the previous step to determine the best preprocessing methodology. Finally, a dendrogram and a heat map were used to illustrate the separability attribute of our dataset. Additionally, we performed a clustering analysis using the K-means algorithm with $\mathrm{k}$ values of one to eleven clusters using all datasets. We plotted the behavior in terms of accuracy and as a confusion matrix.

\section{Supervised learning}

We evaluated the performance of well-known ML classification algorithms, including KNN, SVC, LR, LDA, NB, MLP, RF, and DT. Subsequently, we evaluated DL architectures, such as fully connected neural networks (FNNs) and convolutional neural networks (CNNs).

\section{Neural network architecture}

Two types of networks were used for deep learning; the first is a fully connected neural network and the second is a convolutional neural network. The FNN consists of three fully connected layers of 100 neurons each and the Softsign activation function; then, a final layer of 11 neurons is generated with the sigmoid activation function to generate the probability of the type of cancer. The CNN consists of three convolutional layers with 128 filters each, with a kernel size of 3 and a linear activation function; followed by a layer of 100 fully connected neurons with the Softsign activation function and, finally, a layer of 11 neurons with the Softmax activation function to generate the probability of the type of cancer. Figure 1 shows the architectures used for the experiment, in which the top scheme is a FNN and the bottom scheme is a CNN.

\section{Tuning the algorithms}

Several algorithms were tested by varying or tuning parameter values to find the best performance (Table 2). With these results, we plotted the accuracy values using all datasets created in the training and validation processes and also created confusion matrices. Finally, we did a cross-validation of each algorithm to find the accuracy that was less affected by bias. Additionally, in FNNs and CNNs, we performed a hyperparameter search with a grid search method (GridSearchCV) from the sklearn module, considering the variables shown in Table 3. Due to the high number of parameters, the process of tuning FNNs and CNNs involved choosing 
239 the parameter values that achieved the best accuracy and, then, using these values to find others.

240 The process of finding the best parameter values is presented as follows: 1) batch size and

241 epochs 2) training optimization algorithm 3) learning rate and momentum 4) network weight

242 initialization 5) neuron activation function 6) dropout regularization and 7) number of neurons in

243 the hidden layers.

244

245

246

247

248

\section{Significance Tests}

We performed a test for difference in proportions to determine whether the difference between accuracies of the algorithms is significant. We calculated the differences between the observed

249

250

251

252

253 and expected accuracies under the assumption of a normal distribution. Given the number of correct test predictions $x$ and the number of test instances $N$, accuracy is defined as follows:

$$
\begin{gathered}
A c c_{i}=\frac{x}{N} \\
H_{0}: A c c_{i}-A c c_{j}=0 \\
H_{1}: A c c_{i}-A c c_{j} \neq 0
\end{gathered}
$$

254

255

256

This test allowed determining if the accuracies of the algorithm change significantly after the tuning process and also if there are significant differences between the two algorithms with the highest average accuracies. Based on this, we evaluated whether the parameter tuning of the algorithms was necessary or if the ML algorithm used was more relevant.

259

260

261

262

263

\section{Tools}

264

265

266

267

268

The algorithms were executed using Python programming language and sklearn libraries (Pedregosa et al., 2011), which are explained in (Komer, Bergstra \& Eliasmith, 2014) for ML algorithms. PCA and scaling were executed with decomposition and preprocessing modules from sklearn. Also, DNNs were implemented using Keras (Chollet \& others, 2015). All images were created with matplotlib (Hunter, 2007). The significance tests were performed using R software (Supplementary material 1). The algorithms used here are available at https://github.com/simonorozcoarias/ML_DL_microArrays.

269

270

\section{Results}

271

272

273

\section{Hierarchical analysis}

274

Before evaluating the classification algorithms, we visualized the intrinsic groupings in the data and determined how these groups are influenced by the different preprocessing methodologies

275 applied to our data (Figure 2). Using the downloaded raw data, we created a hierarchical graph

276 (unsupervised learning) using different methodologies (Fig. S1) and concluded that Ward's

277 method produced the most balanced clusters (Figure 3). Then, using only Ward's method, we 
278 performed additional analyses using different datasets, including raw data, scaled data, data

279 transformed by PCA, and data scaled and transformed by PCA. Finally, we created a

280 dendrogram and a heat map to find whether data can be clustered into groups without any given

281 class with the best results. Figure 4 shows four well-separated groups, but the heat map

282 demonstrated other well-conserved groups, which may indicate that the four main clusters could

283 be divided into subgroups.

284

285 Ward's method created four groups, while the other methods clustered the individuals into fewer 286 groups and, in most cases, these groups are largely unbalanced. On the other hand, the raw data

287 and data transformed by PCA performed better in the hierarchical clustering analysis. Employing 288 these datasets, we were able to obtain four and five clusters, respectively. Finally, the heatmaps 289 plotted in Figure 4 showed one group greatly distant from the others (green in Figure 4A and 290 light blue in Figure 4B). On the other hand, the other clusters showed low intra-cluster distances, 291 which is an ideal feature in classification problems (clear blue in Figure 4A and green in Figure 292 4B).

293

294

295

296

297

298

299

300

301

302

303

304

305

306

307

308

309

310

311

312

313

314

315

316

317

Based on a priori knowledge that the number of cancer types is eleven (11), we were interested in determining how the hierarchical clustering algorithm created the cluster assignments.

Therefore, we applied the best parameters found previously (clustering method: ward, and input: raw data and data reduced by PCA). The results shown in Figure 5 and Tables 4 and 5 demonstrate that, although the hierarchical clustering algorithm displays good performance, it does not group the data into the correct number of groups.

Another unsupervised learning assessment involved the implementation of the K-means algorithm. We used all datasets and changed the number of clusters iteratively from one to eleven, increasing by one cluster at a time. Then, we calculated the accuracy in each iteration and a confusion matrix was plotted with the best results (Figure 6). Additionally, we calculated other metrics, such as precision, recall, and f1-score for each class. Overall, the best results were obtained by K-means using 11 clusters with input data processed by PCA, achieving an accuracy of $68.34 \%$ (validation set, using the hold-out splitting method). Also, classes 6, 7 and 9 showed precisions of $100 \%$ and class 5 of $91 \%$ (Table 5).

\section{Algorithm tuning}

The algorithms were tuned by setting several parameters between a given value range (Table 2) to find the best behavior using all datasets. Through this, we aimed to calculate the best hyperparameters for each algorithm and determine which dataset could be the most appropriate. The results of the highest validation accuracies are shown in Table 6. To evaluate overfitting or underfitting, we plotted the accuracy values of the training and validation processes on all datasets described above (Figure 7). RF and DT were not plotted since more than one

Peer) Comput. Sci. reviewing PDF | (CS-2019:09:41248:2:0:NEW 13 Feb 2020) 
318 hyperparameter were tuned. The best results were obtained using $L G$ and raw data. We also 319 calculated a confusion matrix for these results, finding very good classification rates (Figure 8).

320

321

\section{Cross-validation}

322

323

324

325

326

327

328

329

330

331

332

333

334

335

336

337

338

339

340

341

342

343

344

345

346

347

348

349

350

351

352

353

354

355

356

KNN, SVC, LG, MLP, K-MEANS, LDA, NB, RF, and DT were trained and validated with the same fraction of data and each experiment was repeated 10 times to obtain the standard deviations using sklearn's cross-validation function with $\mathrm{k}=10$ (Komer, Bergstra \& Eliasmith, 2014). We used the entire dataset (174) for this procedure. The accuracy and standard deviation results are shown in Table 7.

\section{Deep neural networks}

The grid-search method showed the hyperparameter values that provided the best accuracy in FNN and CNN architectures (Table 8). Figures 9 and 10 show the training results of both architectures, demonstrating how the loss function decreases when most epochs are used until a specific number of epochs is reached ( 80 for FNN and 8 for CNN). Similarly, the accuracy increases in both the training and validation data until reaching the same number of epochs mentioned for the loss function. After this number of epochs, no significant changes were observed for the loss and accuracy values. Using these parameters and cross-validation with $\mathrm{k}=10, \mathrm{FNN}$ and $\mathrm{CNN}$ achieved accuracies of $91.43 \%$ and $94.43 \%$, respectively.

\section{Significance tests}

We performed a test of significant differences, with a $95 \%$ confidence level, between the two best-performing ML algorithms ( $\mathrm{LG}$ and $\mathrm{CNN}$ ). Accordingly, we found no significant differences between the accuracies of these two algorithms ( $p$-value $=0.447$ ).

\section{Discussion}

In this work, we show the application of unsupervised and supervised learning approaches of ML and DL for the classification of 11 cancer types based on a microarray dataset. We observed that the best average results using the training and validation data are obtained using the raw dataset and the Logistic Regression (LR) algorithm, yielding an accuracy value of $100 \%$ (validation set, using the hold-out splitting method). One could assume there is overfitting since the confusion matrix showed an extremely good behavior; however, the comparison of the training and validation accuracies between parameters using the entire dataset may indicate perfect accuracy in both training and validation datasets. Additional tests with independent data should be done to discard potential overfitting. 
357

358

359

360

361

362

363

364

365

366

367

368

369

370

371

372

373

374

375

376

377

378

379

380

381

382

383

384

385

386

387

388

389

390

391

392

393

394

395

396

On the other hand, Multi-Layer Perceptron (MLP) and Linear Discriminant Analysis (LDA) showed a high accuracy value of $97.14 \%$ in the validation dataset. This improvement in accuracy was obtained by optimizing several parameters (number of neurons in MLP) and preprocessing the data set with PCA.

After tuning four parameters, Random forest (RF) obtained high results, with a maximum accuracy of $85.71 \%$. In contrast, Decision trees (DT) obtained 51.14\% accuracy, demonstrating that DT does not work properly for the datasets used in this study, despite tuning several parameters (in our case, three).

Our findings demonstrate that the various algorithms work better by preprocessing the datasets differently. Our results show that MLP, DT, and LDA improved in performance if PCA was applied in advance. However, LG, KNN, NB, RF, and K-means worked better using no preprocessing. Only SVC improved when using scaling and, interestingly, none of the other algorithms showed better results using scaling and PCA on the datasets.

Parameter tuning can improve the accuracy of the algorithm used (Table 7). For instance, SVC obtained a low accuracy of $10.82 \%$ before preprocessing but increased to $81.98 \%$ after tuning. Although most of the algorithms improved their accuracies after the tuning process, only two of them (SVC and K-means) showed significant changes. We conclude that Logistic Regression (LG) is the best ML algorithm for the test dataset in this study, providing an accuracy of $90.6 \%$ with a standard variation of 5.94 from the cross-validation analysis based on ten times. Nevertheless, we recommended using it with moderation. On the other hand, for DL architectures, CNN obtained the best accuracy with 94.43\% (Figure 10). The grid search technique enabled parameter tuning and improved the results, allowing us to propose new DNN architectures (i.e. the architectures showed in Figure 1). Finally, we found no significant difference between the accuracies obtained by LG and CNN.

\section{Conclusions}

Cancer is predicted to become the most deadly disease for humans in the future (Dagenais et al., 2019); therefore, early diagnosis, identification, and treatment are needed to control the disease. ML and DL techniques are promising tools for the classification of cancer types using complex datasets, such as microarrays. In this study, we obtained predictions with as high as 93.52 and $94.46 \%$ accuracies, which will allow patients with these types of pathologies to receive an early and precise detection of their disease, and will also contribute to the discovery of new selective drugs for the treatment of these types of tumors.

\section{References}


397

398

399

400

401

402

403

404

405

406

407

408

409

410

411

412

413

414

415

416

417

418

419

420

421

422

423

424

425

426

427

428

429

430

431

432

433

434

435

436

437

438

439

440

441

442

Alanni R, Hou J, Azzawi H, Xiang Y. 2019. A novel gene selection algorithm for cancer classification using microarray datasets. BMC medical genomics 12:10.

Araújo D, Neto AD, Martins A, Melo J. 2011. Comparative study on dimension reduction techniques for cluster analysis of microarray data. In: The 2011 International Joint Conference on Neural Networks. 1835-1842.

Bengio Y, Courville A, Vincent P. 2013. Representation learning: A review and new perspectives. IEEE Transactions on Pattern Analysis and Machine Intelligence 35:17981828. DOI: 10.1109/TPAMI.2013.50.

Bolón-Canedo V, Sánchez-Marono N, Alonso-Betanzos A, Ben’itez JM, Herrera F. 2014. A review of microarray datasets and applied feature selection methods. Information Sciences 282:111-135.

Ceballos D, López-Álvarez D, Isaza G, Tabares-Soto R, Orozco-Arias S, Ferrin C. 2019. A Machine Learning-based Pipeline for the Classification of CTX-M in Metagenomics Samples. Processes 7:235. DOI: 10.3390/pr7040235.

Chen Y, Li Y, Narayan R, Subramanian A, Xie X. 2016. Gene expression inference with deep learning. Bioinformatics 32:1832-1839.

Chollet F. 2007. Deep Learning with Python. DOI: citeulike-article-id:10054678.

Chollet F, others. 2015. Keras.

Dagenais GR, Leong DP, Rangarajan S, Lanas F, Lopez-Jaramillo P, Gupta R, Diaz R, Avezum A, Oliveira GBF, Wielgosz A, others. 2019. Variations in common diseases, hospital admissions, and deaths in middle-aged adults in 21 countries from five continents (PURE): a prospective cohort study. The Lancet.

Eraslan G, Avsec Ž, Gagneur J, Theis FJ. 2019. Deep learning: new computational modelling techniques for genomics. Nature Reviews Genetics. DOI: 10.1038/s41576-019-0122-6.

Fakoor R, Ladhak F, Nazi A, Huber M. 2013. Using deep learning to enhance cancer diagnosis and classification. In: Proceedings of the international conference on machine learning.

Foss AH, Markatou M, Ray B. 2019. Distance Metrics and Clustering Methods for Mixed-type Data. International Statistical Review 87:80-109.

Géron A. 2017. Hands-on machine learning with Scikit-Learn and TensorFlow: concepts, tools, and techniques to build intelligent systems. "O'Reilly Media, Inc."

Greller LD, Tobin FL. 1999. Detecting selective expression of genes and proteins. Genome Res 9:282-296.

Guillen P, Ebalunode J. 2016. Cancer Classification Based on Microarray Gene Expression Data Using Deep Learning. In: 2016 International Conference on Computational Science and Computational Intelligence Cancer. 208-216. DOI: 10.1109/CSCI.2016.269.

Han D, Kim J. 2018. Unified Simultaneous Clustering and Feature Selection for Unlabeled and Labeled Data. IEEE transactions on neural networks and learning systems 29:6083-6098.

Hunter JD. 2007. Matplotlib: A 2D graphics environment. Computing In Science \& Engineering 9:90-95. DOI: 10.1109/MCSE.2007.55.

Komer B, Bergstra J, Eliasmith C. 2014. Hyperopt-Sklearn: Automatic Hyperparameter Configuration for Scikit-Learn. Scipy 2014:33-39.

Li J, Liu R, Zhang M, Li Y. 2017. Ensemble-based multi-objective clustering algorithms for gene expression data sets. In: 2017 IEEE Congress on Evolutionary Computation (CEC). 333-340.

Liu J, Cai W, Shao X. 2011. Cancer classification based on microarray gene expression data using a principal component accumulation method. Science China Chemistry 54:802-811.

PeerJ Comput. Sci. reviewing PDF | (CS-2019:09:41248:2:0:NEW 13 Feb 2020) 
443

444

445

446

447

448

449

450

451

452

453

454

455

456

457

458

459

460

461

462

463

464

465

466

467

468

469

470

471

472

473

474

475

476

477

478

479

480

481

482

483

484

485

486

487

488

Liu S, Zhang J, Xiang Y, Zhou W, Xiang D. 2019. A Study of Data Pre-processing Techniques for Imbalanced Biomedical Data Classification. arXiv preprint arXiv:1911.00996.

Michie ED, Spiegelhalter DJ, Taylor CC. 1994. Machine Learning, Neural and Statistical Classification. Technometrics 37:459. DOI: 10.2307/1269742.

Min S, Lee B, Yoon S. 2016. Deep learning in bioinformatics. Briefings in bioinformatics:bbw068.

Moosa JM, Shakur R, Kaykobad M, Rahman MS. 2016. Gene selection for cancer classification with the help of bees. BMC medical genomics 9:47.

Motieghader H, Najafi A, Sadeghi B, Masoudi-Nejad A. 2017. A hybrid gene selection algorithm for microarray cancer classification using genetic algorithm and learning automata. Informatics in Medicine Unlocked 9:246-254. DOI: 10.1016/j.imu.2017.10.004.

Orozco-Arias S, Isaza G, Guyot R. 2019. Retrotransposons in Plant Genomes: Structure, Identification, and Classification through Bioinformatics and Machine Learning. International Journal of Molecular Sciences 20:3837. DOI: 10.3390/ijms20153837.

Orozco-arias S, Isaza G, Guyot R, Tabares-soto R. 2019. A systematic review of the application of machine learning in the detection and classi fi cation of transposable elements. Peerj:129. DOI: $10.7717 /$ peerj.8311.

Orozco-Arias S, Núñez-Rincón AM, Tabares-Soto R, López-Álvarez D. 2019. Worldwide cooccurrence analysis of 17 species of the genus Brachypodium using data mining. PeerJ 6:e6193. DOI: 10.7717/peerj.6193.

Pedregosa F, Varoquaux G, Gramfort A, Michel V, Thirion B, Grisel O, Blondel M, Prettenhofer P, Weiss R, Dubourg V, Vanderplas J, Passos A, Cournapeau D, Brucher M, Perrot M, Duchesnay E. 2011. Scikit-learn: Machine Learning in Python. Journal of Machine Learning Research 12:2825-2830.

Perera K, Chan J, Karunasekera S. 2018. Feature Selection for Multiclass Binary Data. In: Pacific-Asia Conference on Knowledge Discovery and Data Mining. 52-63.

Powell WB. 2007. Approximate Dynamic Programming: Solving the curses of dimensionality. John Wiley \& Sons.

Sardana M, Agrawal RK. 2012. A comparative study of clustering methods for relevant gene selection in microarray data. In: Advances in Computer Science, Engineering \& Applications. Springer, 789-797.

Sharma A, Imoto S, Miyano S. 2012. A top-r feature selection algorithm for microarray gene expression data. IEEE/ACM Transactions on Computational Biology and Bioinformatics (TCBB) 9:754-764.

Sirinukunwattana K, Savage RS, Bari MF, Snead DRJ, Rajpoot NM. 2013. Bayesian hierarchical clustering for studying cancer gene expression data with unknown statistics. PloS one 8:e75748.

Statnikov A, Tsamardinos I, Dosbayev Y, Aliferis CF. 2005. GEMS: a system for automated cancer diagnosis and biomarker discovery from microarray gene expression data. International journal of medical informatics 74:491-503.

Su AI, Welsh JB, Sapinoso LM, Kern SG, Dimitrov P, Lapp H, Schultz PG, Powell SM, Moskaluk CA, Frierson HF, Hampton GM. 2001. Molecular classification of human carcinomas by use of gene expression signatures. Cancer Research 61:7388-7393.

Tang Z, Steranka JP, Ma S, Grivainis M, Rodic N, Huang CRL, Shih I-M, Wang T-L, Boeke JD, Fenyo D, Burns KH, Rodić N, Huang CRL, Shih I-M, Wang T-L, Boeke JD, Fenyö D, Burns KH. 2017. Human transposon insertion profiling: Analysis, visualization and

Peer] Comput. Sci. reviewing PDF | (CS-2019:09:41248:2:0:NEW 13 Feb 2020) 
489

490

491

492

493

494

495

496

497

498

499

500

501

502

503

504 identification of somatic LINE-1 insertions in ovarian cancer. PROCEEDINGS OF THE NATIONAL ACADEMY OF SCIENCES OF THE UNITED STATES OF AMERICA 114:E733-E740. DOI: 10.1073/pnas.1619797114.

Varadhachary GR. 2007. Carcinoma of unknown primary origin. Gastrointestinal cancer research: GCR 1:229-35.

Wang Y, Makedon FS, Ford JC, Pearlman J. 2005. HykGene: A hybrid approach for selecting marker genes for phenotype classification using microarray gene expression data. Bioinformatics 21:1530-1537. DOI: 10.1093/bioinformatics/bti192.

Wang S, Wei J. 2017. Feature selection based on measurement of ability to classify subproblems. Neurocomputing 224:155-165.

Wold S, Esbensen K, Geladi P. 1987. Principal component analysis. Chemometrics and intelligent laboratory systems 2:37-52.

Yue T, Wang H. 2018. Deep Learning for Genomics: A Concise Overview. :1-40.

Zou J, Huss M, Abid A, Mohammadi P, Torkamani A, Telenti A. 2018. A primer on deep learning in genomics. Nature Genetics. DOI: 10.1038/s41588-018-0295-5. 
Figure 1

Artificial neural network architectures used for cancer classification.

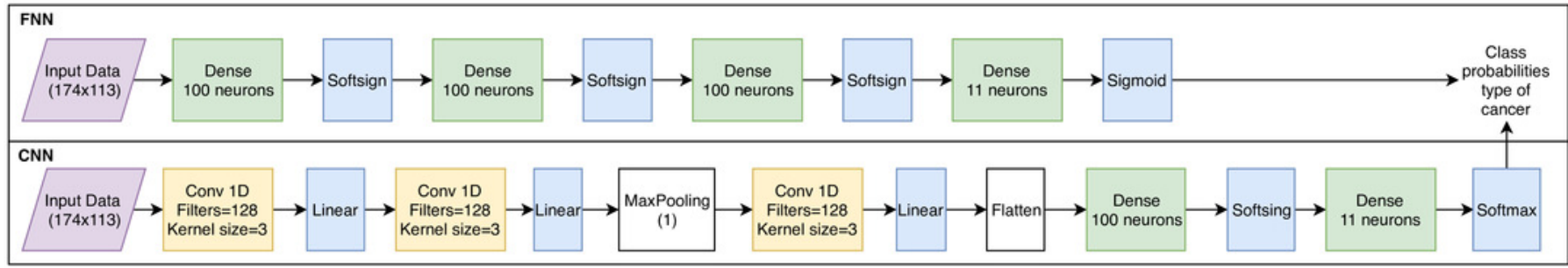


Figure 2

Hierarchical maps using Ward as clustering method and A) raw data B) scaled data, C) data reduced by PCA and D) data scaled and reduced by PCA.

Due to the large number of characteristics of the data set, it is recommended that you transform the data set to use only the most relevant and informative variables, which is called the preprocessing step.

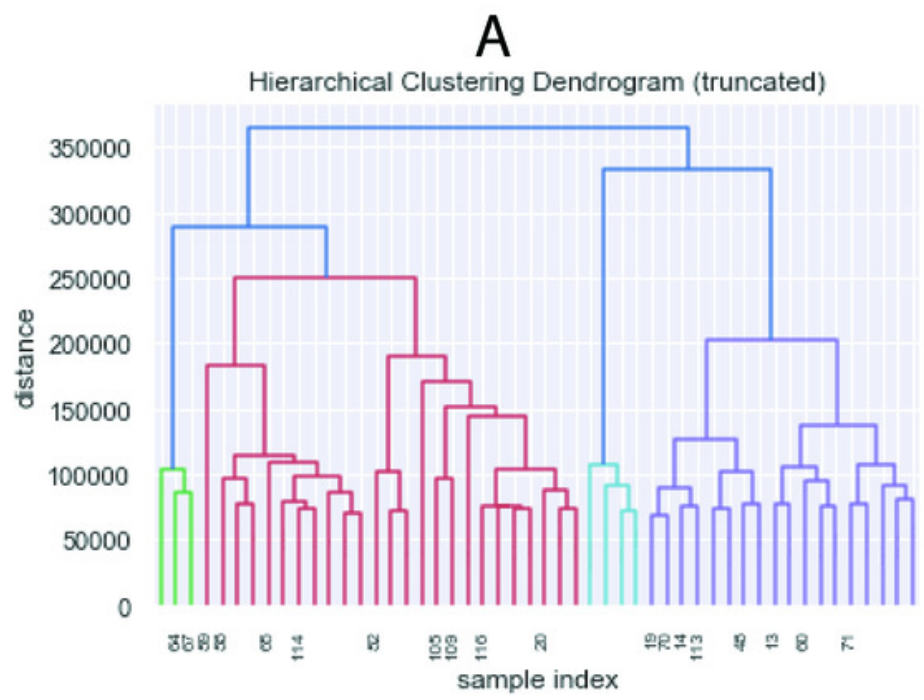

C

Hierarchical Clustering Dendrogram (truncated)

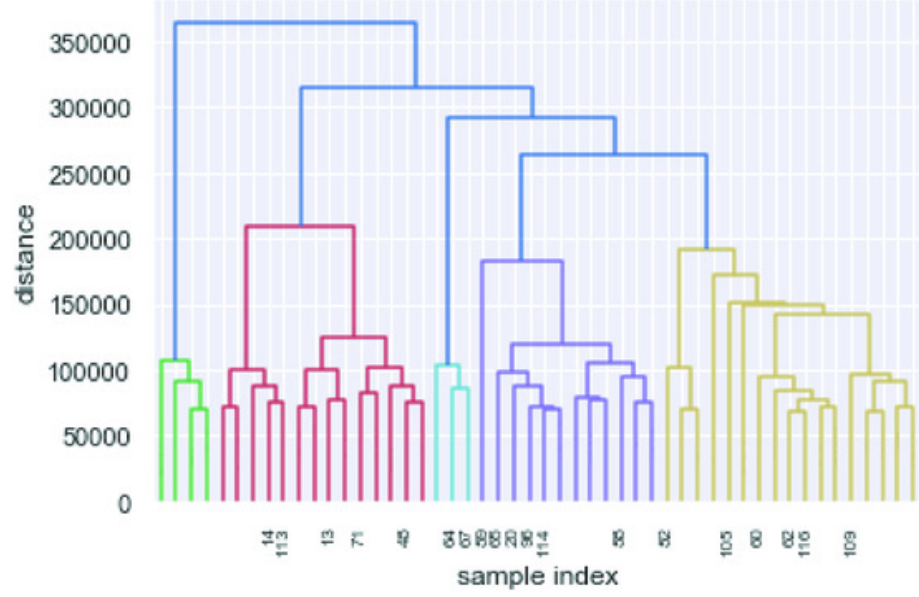

B

Hierarchical Clustering Dendrogram (truncated)

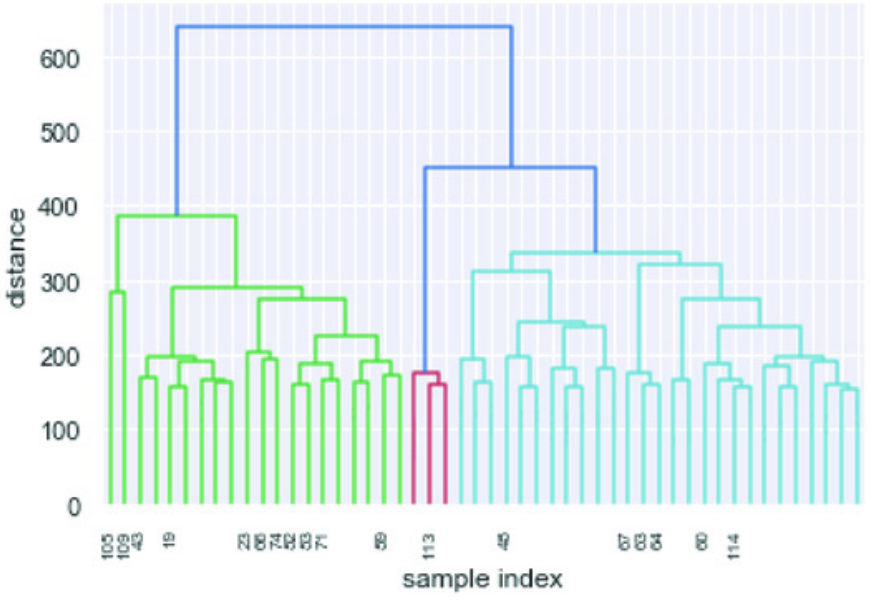

D

Hierarchical Clustering Dendrogram (truncated)

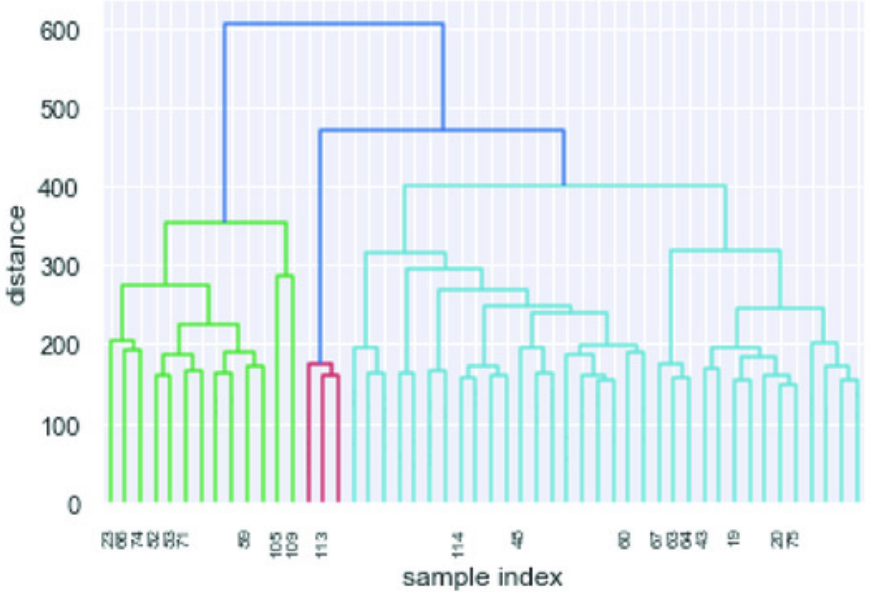




\section{Figure 3}

Hierarchical maps using Ward method as the criterion for choosing the pair of clusters to merge at each step.

This hierarchical map was generated by data without transformation and deleting their labels. Clustering approaches demonstrate whether the data contain relevant patterns for grouping.

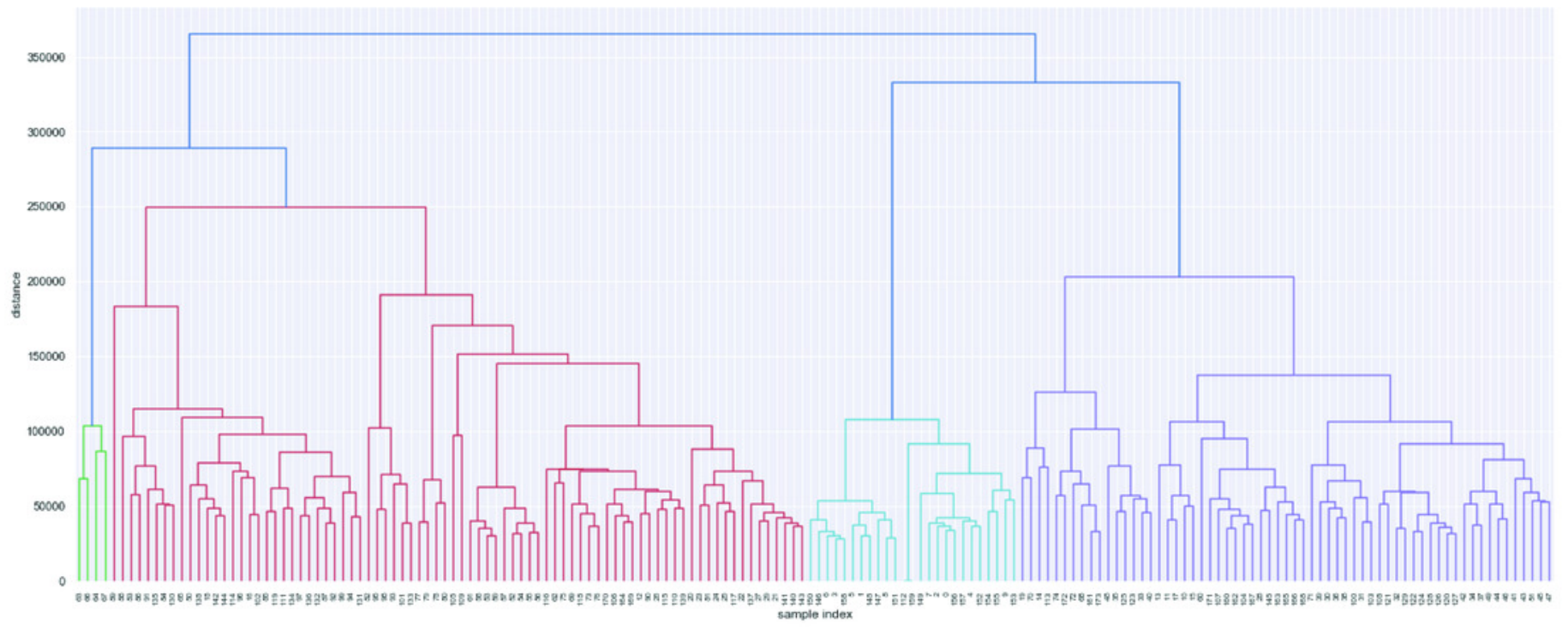


Figure 4

Hierarchical and heat map analysis utilizing A) raw data and B) Data processed by PCA.

These heat maps show how similar (near zero) or different (about 200,000) the individuals in the clusters are. A cluster is interesting when its members are very similar and are very different from individuals in other groups.

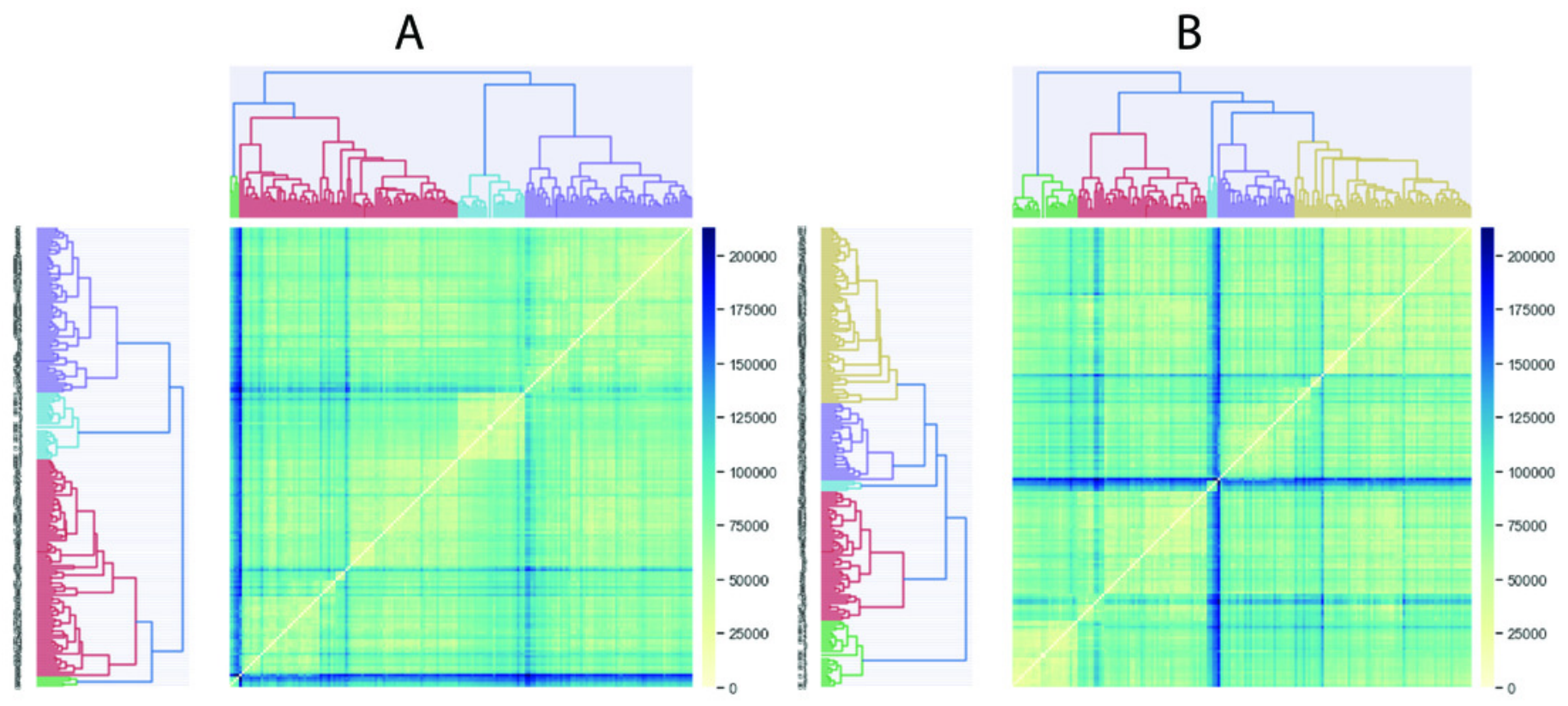




\section{Figure 5}

Clusters composition using A) raw data and B) Data processed by PCA.

Clustering was performed using Ward as distance algorithm. Label correspond to the cluster number predicted by the algorithm and may not correspond to labels of Table 1 . 


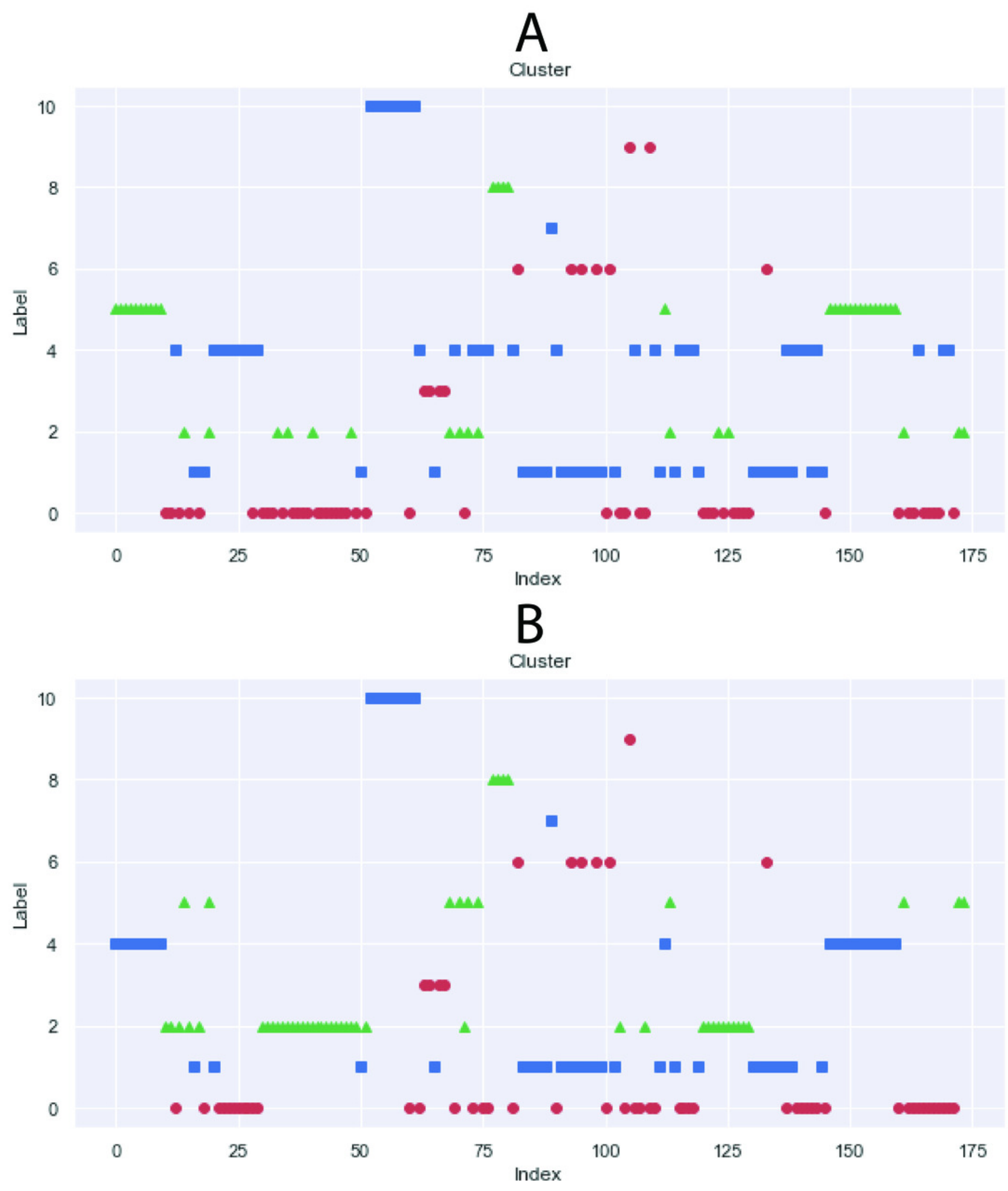




\section{Figure 6}

A) Behavior of Accuracy in terms of number of clusters and B) Confusion matrix with best results (clusters $=11$ ) using $\mathrm{K}$-means algorithm.

Results showed in 6 A are the accuracy using validation data set which correspond to $20 \%$ of whole data.

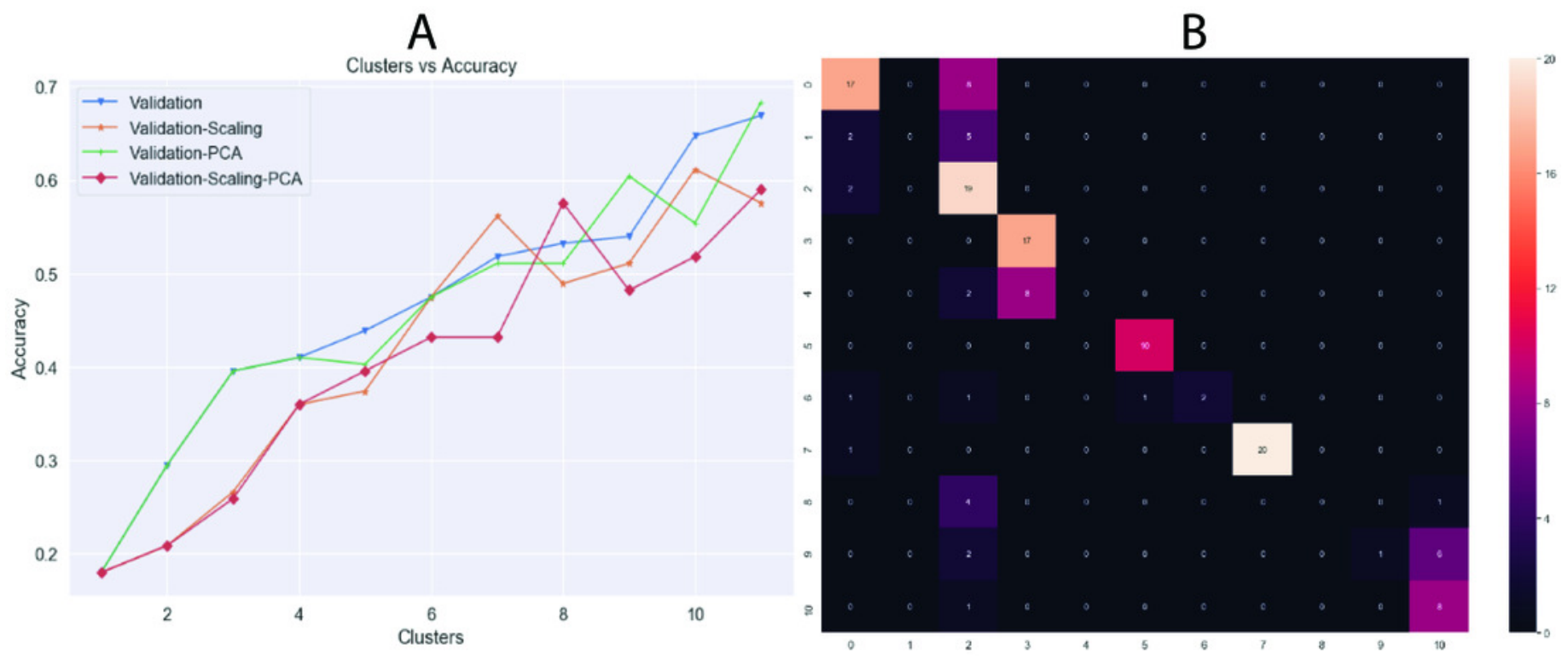




\section{Figure 7}

Comparison of training and validation accuracy between parameters using all data set and A) KNN, B) SVC, C) LG, D) MLP, and E) K-Means.

Algorithm do not present in this Figure, appear in Table 5 as default in column "Tuned Parameters". 
A

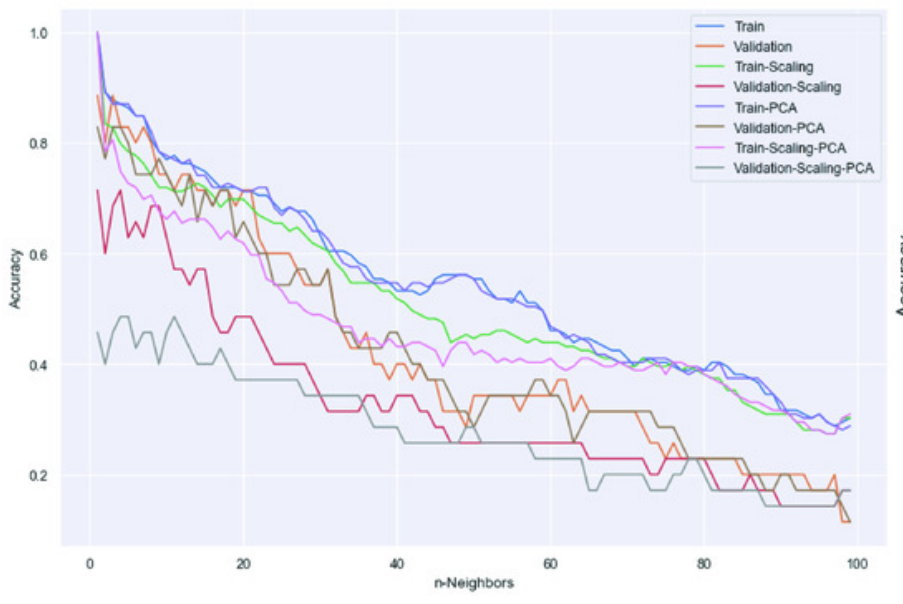

C

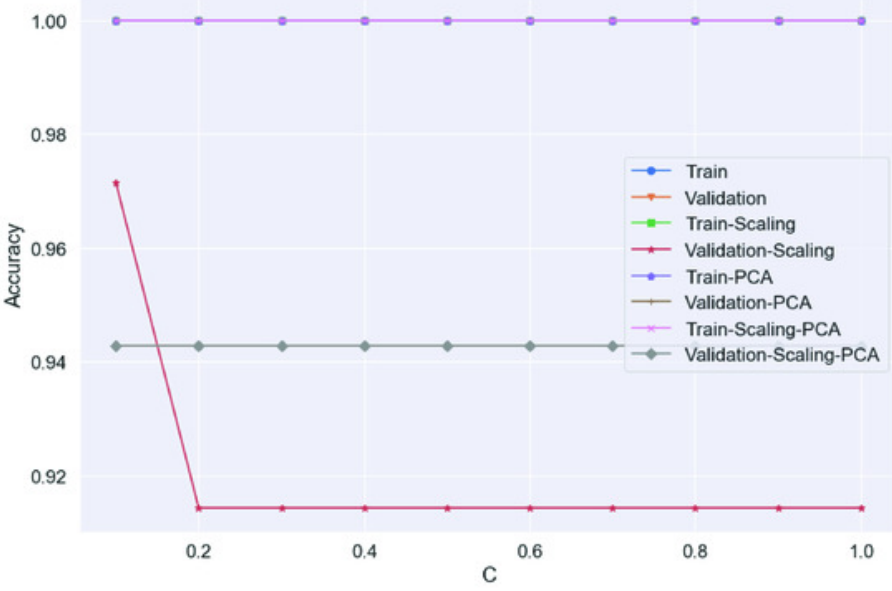

$\mathrm{E}$

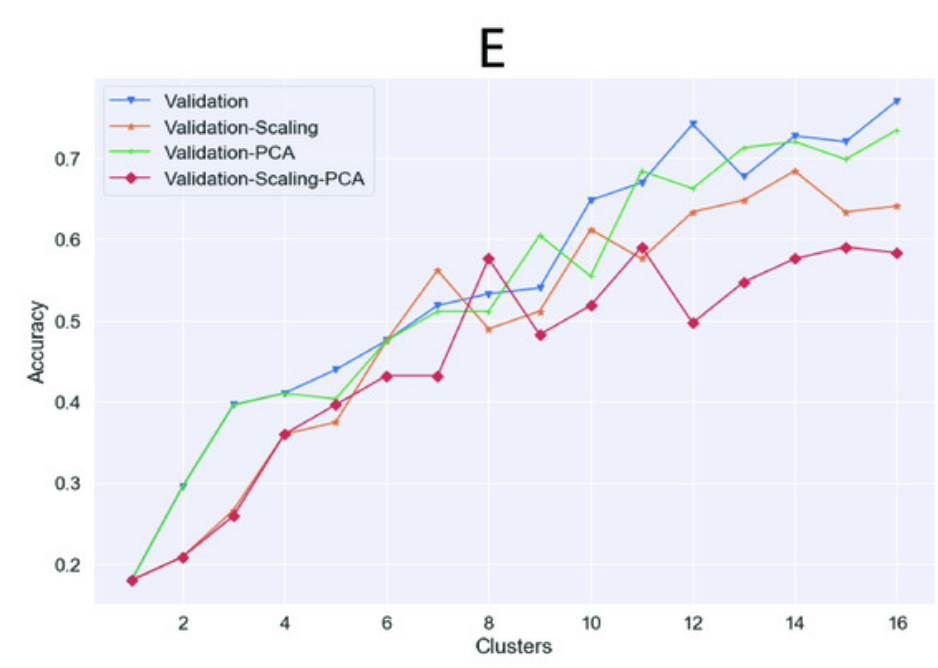

B

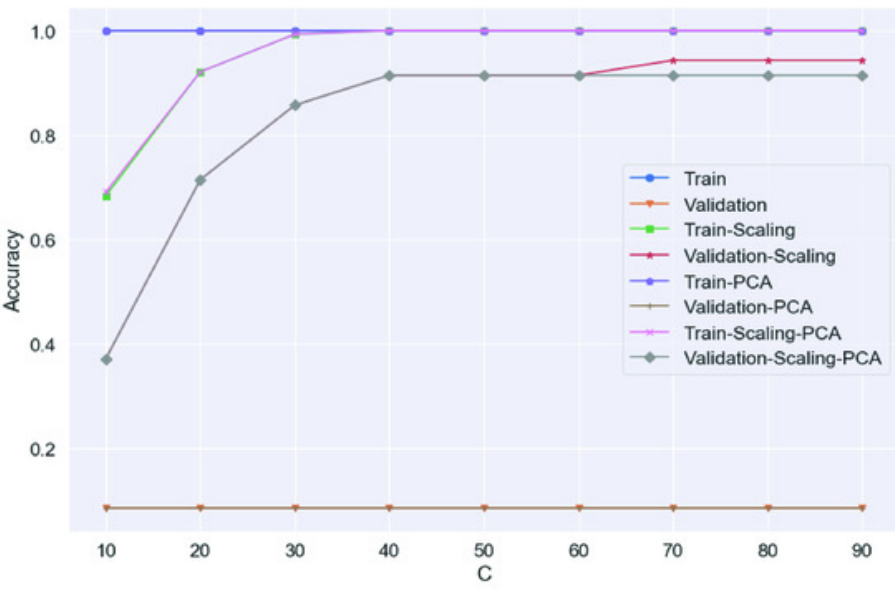

D

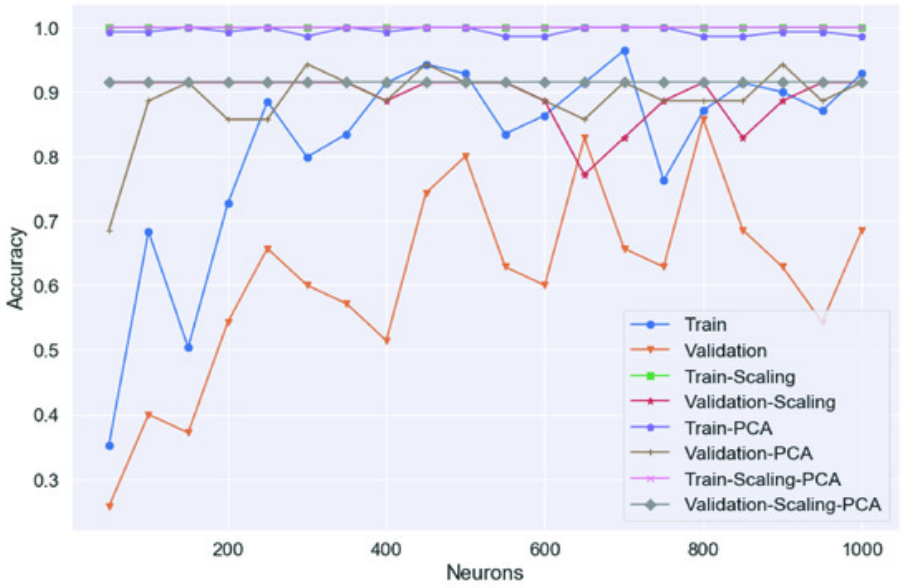


Figure 8

Confusion matrix of $L G$ algorithm results.

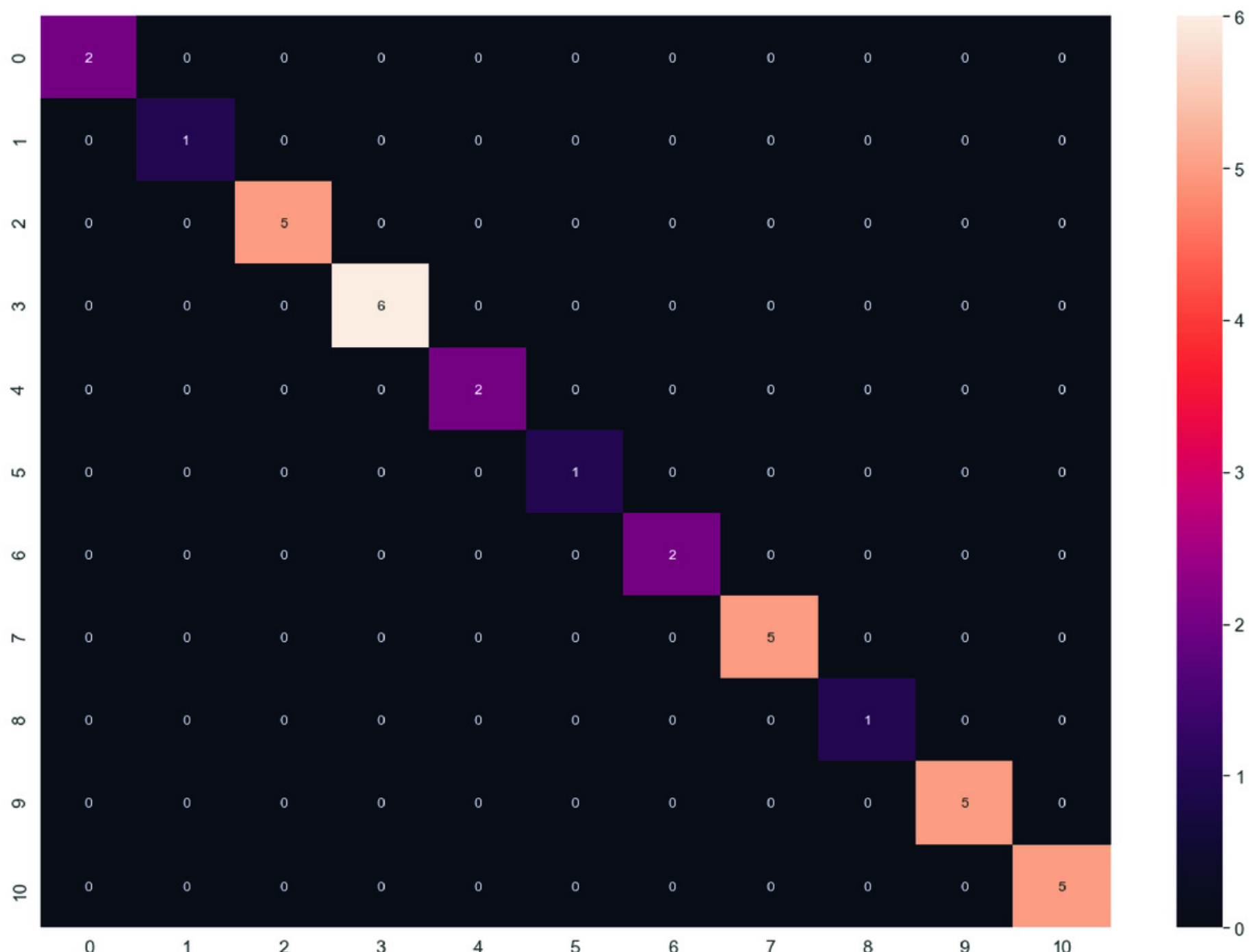


Figure 9

Results obtained by FNN architecture in training using 100 epochs.

A) Lost value and B) Accuracy. Lost function and accuracy is plotted on both training and validation data sets in order to observe behavior. When both data sets show very distant results, the architecture may be overfitting.
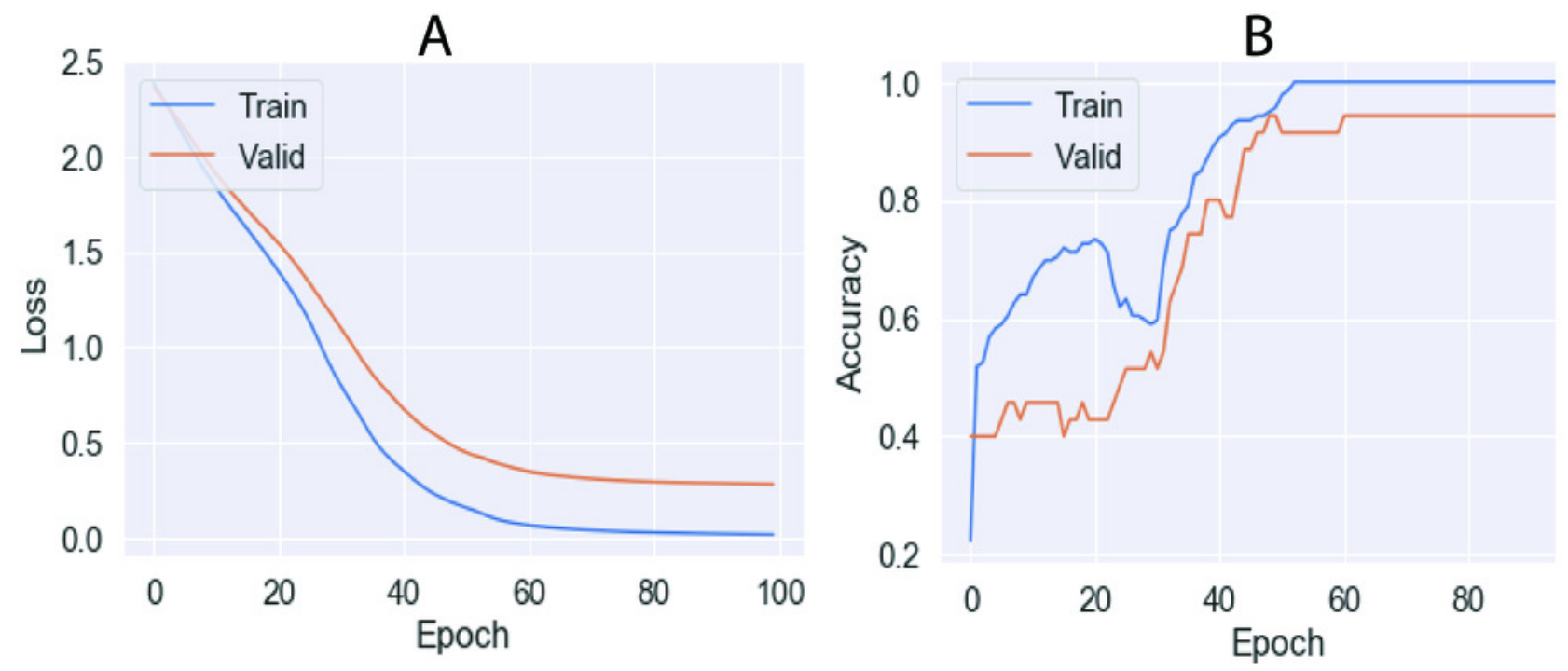


\section{Figure 10}

Results obtained by CNN architecture in training using 10 epochs.

A) Lost value and B) Accuracy. Lost function and accuracy is plotted on both training and validation data sets in order to observe behavior. When both data sets show very distant results, the architecture may be overfitting.
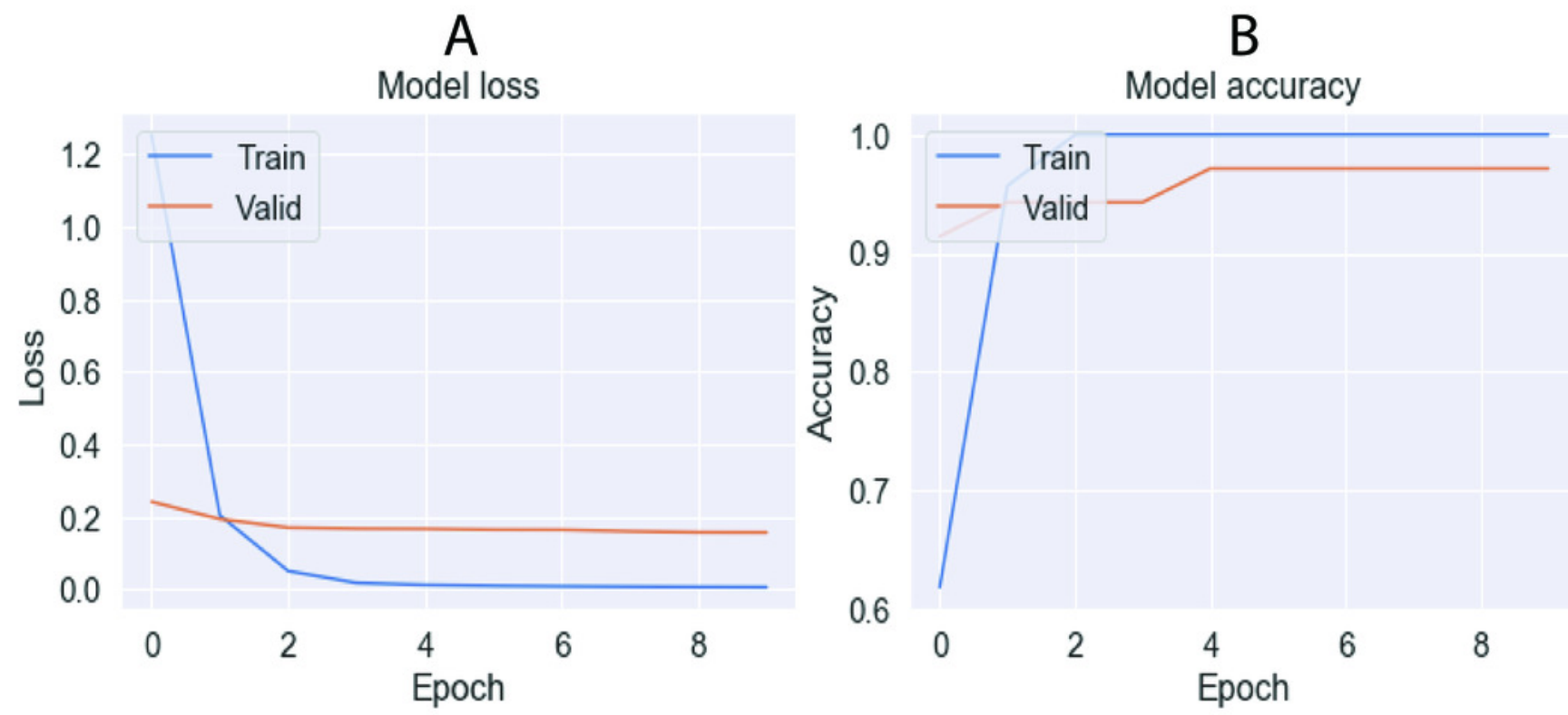


\section{Table $\mathbf{1}$ (on next page)}

Cancer type classification in the 11_tumor data base. 


\begin{tabular}{|c|c|c|}
\hline Class & Cancer type & $\begin{array}{c}\text { number of } \\
\text { patients }\end{array}$ \\
\hline 0 & Ovary & 27 \\
\hline 1 & Bladder/Ureter & 8 \\
\hline 2 & Breast & 26 \\
\hline 3 & Colorectal & 23 \\
\hline 4 & gastroesophagus & 12 \\
\hline 5 & Kidney & 11 \\
\hline 6 & Liver & 7 \\
\hline 7 & Prostate & 26 \\
\hline 8 & Pancreas & 6 \\
\hline 9 & adenocarcinoma & 14 \\
\hline 10 & $\begin{array}{c}\text { lung squamous } \\
\text { cell carcinoma }\end{array}$ \\
\hline
\end{tabular}




\section{Table 2 (on next page)}

Tested Algorithm Parameters. 


\begin{tabular}{|c|c|c|c|c|}
\hline $\begin{array}{l}\text { Algorith } \\
\mathrm{m}\end{array}$ & Parameter & Range & Step & Description \\
\hline$K N N$ & n_neighbors & $1-99$ & 1 & $\begin{array}{l}\text { Number of } \\
\text { neighbors }\end{array}$ \\
\hline$S V C$ & $\mathrm{C}$, gamma & 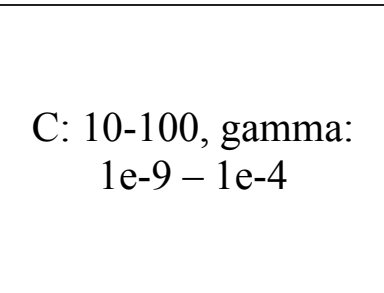 & $\begin{array}{c}\mathrm{C}: 10 \\
\text { gamma: } \\
10\end{array}$ & $\begin{array}{l}\text { Penalty parameter } \\
\text { C of the error term. } \\
\text { Gamma is the free } \\
\text { parameter of the } \\
\text { Gaussian radial } \\
\text { basis function }\end{array}$ \\
\hline$L G$ & $\mathrm{C}$ & $0.1-1$ & 0.1 & $\begin{array}{c}\text { Inverse of } \\
\text { regularization } \\
\text { strength } \\
\end{array}$ \\
\hline$L D A$ & $\mathrm{~N} / \mathrm{A}$ & $\mathrm{N} / \mathrm{A}$ & $\mathrm{N} / \mathrm{A}$ & N/A \\
\hline$N B$ & $\mathrm{~N} / \mathrm{A}$ & $\mathrm{N} / \mathrm{A}$ & $\mathrm{N} / \mathrm{A}$ & $\mathrm{N} / \mathrm{A}$ \\
\hline$M L P$ & $\begin{array}{c}\text { solver='lbfgs', } \\
\text { alpha=0.5, } \\
\text { hidden_layer_sizes }\end{array}$ & $50-1050$ & 50 & $\begin{array}{c}\text { Number of } \\
\text { neurons in hidden } \\
\text { layers. In this } \\
\text { study we used } \\
\text { solver lbfgs and } \\
\text { alpha } 0.5\end{array}$ \\
\hline$R F$ & $\begin{array}{c}\text { n_estimators, } \\
\text { max_depth, } \\
\text { min_samples_split, } \\
\text { max_features }\end{array}$ & $\begin{array}{l}\text { n_estimators: } 1 \text { - } 91, \\
\text { max_depth: } 1-91, \\
\text { min_samples_split: } \\
10-100, \\
\text { max_features: } 10-90\end{array}$ & $\begin{array}{l}10 \text { for all } \\
\text { paramete } \\
\text { rs }\end{array}$ & N/A \\
\hline$D T$ & $\begin{array}{c}\text { max_depth, } \\
\text { min_samples_split, } \\
\text { max_features }\end{array}$ & $\begin{array}{l}\text { max_depth: } 1-91, \\
\text { min_samples_split: } \\
\text { 10 - 100, } \\
\text { max_features: } 10-90\end{array}$ & $\begin{array}{l}10 \text { for all } \\
\text { paramete } \\
\text { rs }\end{array}$ & N/A \\
\hline $\begin{array}{c}K- \\
\text { Means }\end{array}$ & $\begin{array}{c}\text { n_clusters, } \\
\text { random_state }=0\end{array}$ & $1-17$ & 1 & $\begin{array}{l}\text { Number of } \\
\text { clusters. In this } \\
\text { study we used } \\
\text { random state } \\
\text { equals to zero. }\end{array}$ \\
\hline
\end{tabular}




\section{Table 3(on next page)}

Parameters tuned in DNNs. 


\begin{tabular}{|c|c|c|}
\hline Parameter & Values & Description \\
\hline Batch size & $\begin{array}{l}10, \quad 20,30, \quad 40,50, \quad 60,70, \\
80,90,100\end{array}$ & $\begin{array}{l}\text { Number of training examples } \\
\text { utilized in one iteration }\end{array}$ \\
\hline Epochs & $10,50,100,200$ & $\begin{array}{l}\text { Number of times that the } \\
\text { learning algorithm will work } \\
\text { through the entire training }\end{array}$ \\
\hline $\begin{array}{l}\text { Training } \quad \text { Optimization } \\
\text { Algorithm }\end{array}$ & $\begin{array}{l}\text { SGD, RMSprop, Adagrad, } \\
\text { Adadelta, Adam, Adamax, } \\
\text { Nadam }\end{array}$ & $\begin{array}{l}\text { tools that update model } \\
\text { parameters and minimize the } \\
\text { value of the loss function, as } \\
\text { evaluated on the training set }\end{array}$ \\
\hline Learning Rate & $0.001,0.01,0.1,0.2,0.3$ & $\begin{array}{l}\text { Hyper-parameter } \\
\text { controls how much the } \\
\text { weights are being adjusting } \\
\text { with respect to the loss } \\
\text { gradient }\end{array}$ \\
\hline Momentum & $0.0,0.2,0.4,0.6,0.8,0.9$ & $\begin{array}{l}\text { Value between } 0 \text { and } 1 \text { that } \\
\text { increases the size of the steps } \\
\text { taken towards the minimum } \\
\text { by trying to jump from a } \\
\text { local minima }\end{array}$ \\
\hline $\begin{array}{l}\text { Network } \\
\text { Initialization }\end{array}$ & $\begin{array}{l}\text { uniform, lecun_uniform, } \\
\text { normal, zero, glorot_normal, } \\
\text { glorot_uniform, he_normal, } \\
\text { he_uniform }\end{array}$ & $\begin{array}{l}\text { Initialization of weights into } \\
\text { hidden layers of the network. }\end{array}$ \\
\hline Neuron Activation Function & $\begin{array}{l}\text { softmax, softplus, softsign, } \\
\text { relu, tanh, sigmoid, } \\
\text { hard_sigmoid, linear }\end{array}$ & $\begin{array}{l}\text { How the neuron output is } \\
\text { activated based on its inputs }\end{array}$ \\
\hline Dropout Regularization & $\begin{array}{l}0.0,0.1,0.2,0.3,0.4,0.5,0.6 \\
0.7,0.8,0.9\end{array}$ & $\begin{array}{l}\text { Process of randomly } \\
\text { dropping out nodes during } \\
\text { training }\end{array}$ \\
\hline Weight constraint & $1,2,3,4,5$ & $\begin{array}{l}\text { Value that introduces a } \\
\text { penalty to the loss function } \\
\text { when training a neural } \\
\text { network to encourage the } \\
\text { network to use small weights }\end{array}$ \\
\hline $\begin{array}{l}\text { Number of Neurons in the } \\
\text { Hidden Layers }\end{array}$ & $\begin{array}{l}1,5,10,20,30,40,50,60 \\
70,80,90,100\end{array}$ & $\begin{array}{l}\text { Amount of neurons that } \\
\text { composed each hidden layers } \\
\text { of the network. }\end{array}$ \\
\hline
\end{tabular}




\section{Table 4 (on next page)}

Cluster composition and original number of individuals from each class of cancer. 


\begin{tabular}{|r|c|c|c|}
\hline \multicolumn{1}{|c|}{ Class } & $\begin{array}{l}\text { Original } \\
\text { number }\end{array}$ & $\begin{array}{l}\text { Clustering using raw } \\
\text { data }\end{array}$ & $\begin{array}{l}\text { Clustering using data } \\
\text { processed by PCA }\end{array}$ \\
\hline 0 & 27 & 47 & 47 \\
\hline 1 & 8 & 29 & 28 \\
\hline 2 & 26 & 16 & 39 \\
\hline 3 & 23 & 4 & 25 \\
\hline 4 & 12 & 31 & 10 \\
\hline 5 & 11 & 25 & 6 \\
\hline 6 & 7 & 6 & 4 \\
\hline 7 & 26 & 1 & 1 \\
\hline 8 & 6 & 4 & 9 \\
\hline 9 & 14 & 2 & \\
\hline 10 & 14 & 9 & \\
\hline
\end{tabular}




\section{Table 5 (on next page)}

Metrics obtained by K-Means for each cancer type. 


\begin{tabular}{|r|c|c|c|}
\hline \multicolumn{1}{|c|}{ Class } & Precision & Recall & F1-Score \\
\hline 0 & 0.74 & 0.68 & 0.71 \\
\hline 1 & 0 & 0 & 0 \\
\hline 2 & 0.45 & 0.9 & 0.6 \\
\hline 3 & 0.68 & 1 & 0.81 \\
\hline 4 & 0 & 0 & 0 \\
\hline 5 & 0.91 & 1 & 0.95 \\
\hline 6 & 1 & 0.4 & 0.57 \\
\hline 7 & 1 & 0.95 & 0.98 \\
\hline 8 & 0 & 0 & 0 \\
\hline 9 & 1 & 0.11 & 0.2 \\
\hline 10 & 0.53 & 0.89 & 0.67 \\
\hline
\end{tabular}




\section{Table 6(on next page)}

Tuning hyperparameters of best results of algorithms tested. 


\begin{tabular}{|c|c|c|c|}
\hline \multicolumn{4}{|c|}{ Results on validation data (The best result) } \\
\hline Algorithm & $\begin{array}{c}\text { Conditions on the } \\
\text { data set }\end{array}$ & Tuning parameters & $\begin{array}{c}\% \\
\text { Accuracy } \\
\end{array}$ \\
\hline \multirow{4}{*}{ KNN } & Any & Neighbors $=1$ & 88.57 \\
\hline & Scaling & Neighbors: 1 & 71.43 \\
\hline & PCA & Neighbors: 1 & 82.86 \\
\hline & Scaling + PCA & Neighbors: 4 & 48.57 \\
\hline \multirow{4}{*}{ SVC } & Any & $\mathrm{C}=10$ & 8.57 \\
\hline & Scaling & $\mathrm{C}=70$ & 94.29 \\
\hline & PCA & $\mathrm{C}=10$ & 8.57 \\
\hline & Scaling + PCA & $\mathrm{C}=40$ & 91.43 \\
\hline \multirow{4}{*}{$\begin{array}{l}\text { Logistic } \\
\text { Regression }\end{array}$} & Any & $\mathrm{C}=0,1$ & 100.00 \\
\hline & Scaling & $\mathrm{C}=0,1$ & 97.14 \\
\hline & PCA & $\mathrm{C}=0,1$ & 94.29 \\
\hline & Scaling + PCA & $\mathrm{C}=0,1$ & 94.29 \\
\hline \multirow{4}{*}{$\begin{array}{l}\text { Linear } \\
\text { Discrimina } \\
\text { nt Analysis }\end{array}$} & Any & Default & 91.43 \\
\hline & Scaling & Default & 91.43 \\
\hline & PCA & Default & 97.14 \\
\hline & Scaling + PCA & Default & 82.86 \\
\hline \multirow{4}{*}{$\begin{array}{c}\text { Gaussian } \\
\text { NB }\end{array}$} & Any & Default & 85.71 \\
\hline & Scaling & Default & 85.71 \\
\hline & PCA & Default & 80.00 \\
\hline & Scaling + PCA & Default & 71.43 \\
\hline \multirow{4}{*}{$\begin{array}{l}\text { Random } \\
\text { Forest }\end{array}$} & Any & $\begin{array}{l}\text { n_estimators }=81, \\
\text { max_depth }=91, \\
\text { min_samples_split }= \\
10, \text { max_features }=50\end{array}$ & 97.14 \\
\hline & Scaling & $\begin{array}{l}\text { n_estimators }=91, \\
\text { max_depth }=81, \\
\text { min_samples_split }= \\
10, \text { max_features }=60\end{array}$ & 97.14 \\
\hline & PCA & $\begin{array}{l}\text { n_estimators }=91, \\
\text { max_depth }=21, \\
\text { min_samples_split= } \\
10, \text { max_features }=30\end{array}$ & 94.28 \\
\hline & Scaling + PCA & $\begin{array}{l}\text { n_estimators }=61, \\
\text { max_depth }=11, \\
\text { min_samples_split }= \\
10, \text { max_features }=20\end{array}$ & 85.71 \\
\hline \multirow{2}{*}{$\begin{array}{l}\text { Decision } \\
\text { Tree }\end{array}$} & Any & $\begin{array}{l}\text { max_depth }=71, \\
\text { min_samples_split }= \\
10, \text { max_features }=40\end{array}$ & 68.57 \\
\hline & Scaling & $\begin{array}{l}\text { max_depth }=51, \\
\text { min_samples_split }= \\
10, \text { max_features }=60\end{array}$ & 68.57 \\
\hline
\end{tabular}




\begin{tabular}{|c|c|c|c|} 
& PCA & $\begin{array}{l}\text { max_depth=81, } \\
\text { min_samples_split= } \\
\text { 10,_max_features=30 }\end{array}$ & 82.85 \\
\cline { 2 - 4 } & Scaling + PCA & $\begin{array}{l}\text { max_depth=51, } \\
\text { min_samples_split= } \\
\text { 20, max_features=60 }\end{array}$ & 74.28 \\
\hline \multirow{3}{*}{$\begin{array}{c}\text { Multi- } \\
\text { Layer } \\
\text { Perceptron }\end{array}$} & Any & Neurons=800 & 85.71 \\
\cline { 2 - 4 } & Scaling & Neurons=50 & 91.43 \\
\cline { 2 - 4 } & PCA & Neurons=300 & 97.14 \\
\cline { 2 - 4 } & Scaling + PCA & Neurons=50 & 91.43 \\
\cline { 2 - 4 } K-Means & Any & Clusters=16 & 76.97 \\
\cline { 2 - 4 } & Scaling & Clusters=14 & 68.34 \\
\cline { 2 - 4 } & PCA & Clusters=16 & 73.38 \\
\hline
\end{tabular}




\section{Table 7 (on next page)}

Cross validation of KNN, SVC, LG, MLP, K-Means, LDA, NB, RF and DT before and after of tuning process. 


\begin{tabular}{|c|c|c|c|c|c|}
\hline \multicolumn{6}{|c|}{ Results of Cross Validation (10 splits) } \\
\hline & \multicolumn{2}{|c|}{ Before Tuning } & \multicolumn{2}{|c|}{ After Tuning } & \multirow{2}{*}{$\begin{array}{c}\text { Significance } \\
\text { difference }\end{array}$} \\
\hline Algorithm & $\begin{array}{c}\% \\
\text { accuracy }\end{array}$ & $\begin{array}{l}\text { Standard } \\
\text { Deviation }\end{array}$ & $\begin{array}{c}\% \\
\text { accuracy }\end{array}$ & $\begin{array}{l}\text { Standard } \\
\text { Deviation }\end{array}$ & \\
\hline $\mathrm{KNN}$ & 78.3 & 12.71 & 82.03 & 10.19 & $\mathrm{NO}$ \\
\hline SVC & 10.82 & 6.65 & 81.98 & 13.7 & YES \\
\hline Logistic Regression & 90.6 & 7.93 & 90.6 & 5.94 & $\mathrm{NO}$ \\
\hline $\begin{array}{l}\text { Multi-Layer } \\
\text { Perceptron }\end{array}$ & 79.89 & 20.62 & 83.40 & 13.64 & $\mathrm{NO}$ \\
\hline K-Means & 10.16 & 9.36 & 68.34 & 9.26 & YES \\
\hline $\begin{array}{c}\text { Linear Discriminant } \\
\text { Analysis }\end{array}$ & 83.4 & 11.62 & $\mathrm{~N} / \mathrm{A}$ & $\mathrm{N} / \mathrm{A}$ & N/A \\
\hline Gaussian NB & 84.12 & 12.78 & $\mathrm{~N} / \mathrm{A}$ & $\mathrm{N} / \mathrm{A}$ & $\mathrm{N} / \mathrm{A}$ \\
\hline Random Forest & 66.75 & 13.79 & 72.69 & 15.85 & $\mathrm{NO}$ \\
\hline Decision Tree & 69.78 & 14.9 & 66.04 & 15.45 & $\mathrm{NO}$ \\
\hline
\end{tabular}




\section{Table 8 (on next page)}

Best value of hyperparameters tuned in deep neural networks. 


\begin{tabular}{|l|c|c|}
\hline & \multicolumn{2}{|c|}{ Best Value } \\
\hline Parameter & FNN & CNN \\
\hline Batch size & 20 & 10 \\
\hline Epochs & 100 & 10 \\
\hline $\begin{array}{l}\text { Training optimization } \\
\text { algorithm }\end{array}$ & Adagrad & SGD \\
\hline Learn rate & 0.2 & 0.1 \\
\hline Momentum & 0 & 0 \\
\hline $\begin{array}{l}\text { Network Weight } \\
\text { Initialization }\end{array}$ & normal & glorot_normal \\
\hline $\begin{array}{l}\text { Neuron Activation } \\
\text { Function }\end{array}$ & softsign & linear \\
\hline Weight constraint & 3 & 1 \\
\hline Dropout Regularization & 0 & 0.4 \\
\hline
\end{tabular}

